\title{
Leg Telescopic Folding Table Using Servomotors
}

\author{
Takaaki Ikeda, Yhuki Kitazono \\ National Institute of Technology, Kitakyusyu College, 5-20-1 Shii, Kokuraminami-ku, Kitakyusyu-city, Fukuoka \\ 802-0985, Japan
}

*Corresponding Author: kitazono@kct.ac.jp

\begin{abstract}
The current population of disabled persons is not small, and all disabled persons have all sorts of obstacles in their lives, and there are many useful things to eliminate them. For example, there are convenient things such as automatic wheelchairs made for people who can't move their legs, traffic lights with voice for people with disabilities, and automatic eating robots for people with disabilities. There are many useful things such as self-opening doors and electric bicycles for the elderly and the general public as well as physically disabled people.

Under such situations, what things are useful for disabled people and the elderly? In this research, servomotor, Arduino was used to make things useful for disabled people and the elderly
\end{abstract}

Keywords: motor, servomotor, Arduino.

\section{Introduction}

There are two types of tables. One is used indoors ${ }^{(1)}$ and the other is a table used outside. In the case of an indoor table, it will not be moved a lot of times in the living room. In the case of a table used outside, it is necessary to carry around and most things need to assemble.

So we focused on assembling / folding work. Currently carried tables is easily assembled and used by many people. However, most things are often used by both hands to fold and assemble. Also, large-sized tables used in camps and school sports festivals, etc. are heavy and more hard to assemble and folding. Those can be handled comfortably by most people, but people with disabilities, small children and elderly people are hard to handle.

In addition, disabled people increase year by year ${ }^{(2)}$, the population of young people is decreasing and the number of elderly people is increasing ${ }^{(3)}$. For that reason, I felt the necessity of convenient and items, and I decided to study the carried table.

Many tables used outside have fixed legs, and most tables used inside a house or in a room are also fixed. The table should be placed on a stable ground to put things on its own. In this case, the table used outside may not be used on a stable ground, so in this paper we attached a motor to each leg to stand stably on unstable ground.

\section{System structure}

The overall structure is shown in Fig 1. The system uses two DC power supplies, two Arduino UNOs, and an Arduino MEGA, and three TA7291Ps as motor drivers. This system consists of a table and a sensor.

As can be seen from Fig. 1, this system has servomotors attached to the legs of the table, and this servomotor is a joint for the legs and is perform the folding operation, and as shown in Fig. 2. The leg uses a motor, and a tire is attached to the tip of the leg. This motor is for extending and retracting the body of the leg. The central part of the table is a node at the time of folding, and as shown in Fig. 1, the servomotor is also attached to this part. And four tires are attached to the end of the table.

The sensor unit consists of Arduino and Arduino MEGA and a switch. The movement of the table is controlled by Arduino and Arduino MEGA.

Two current sources are used from the general view of Fig 1. This was attached to move the three motors which attached to the four legs of the table respectively. Only three motors move with respect to the four legs. This is because one is at the standard height. 


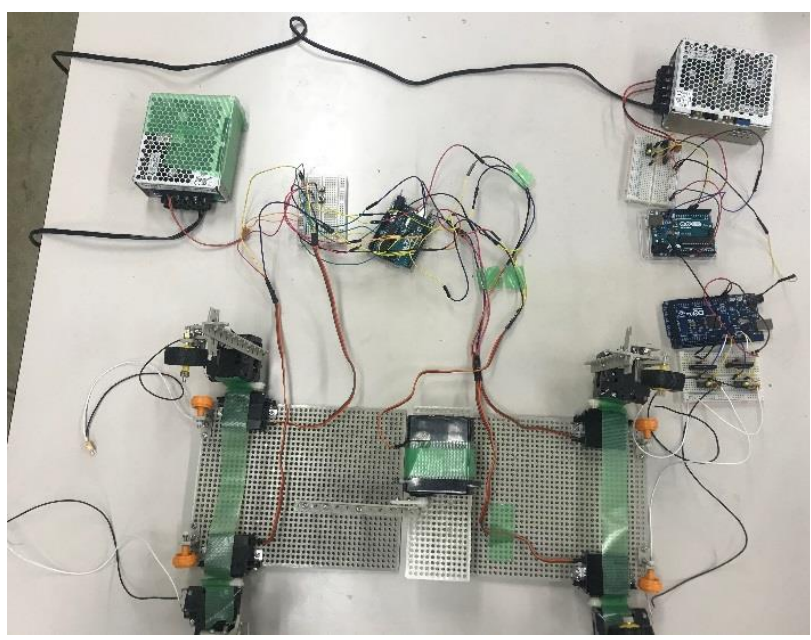

Fig. 1. Overall view

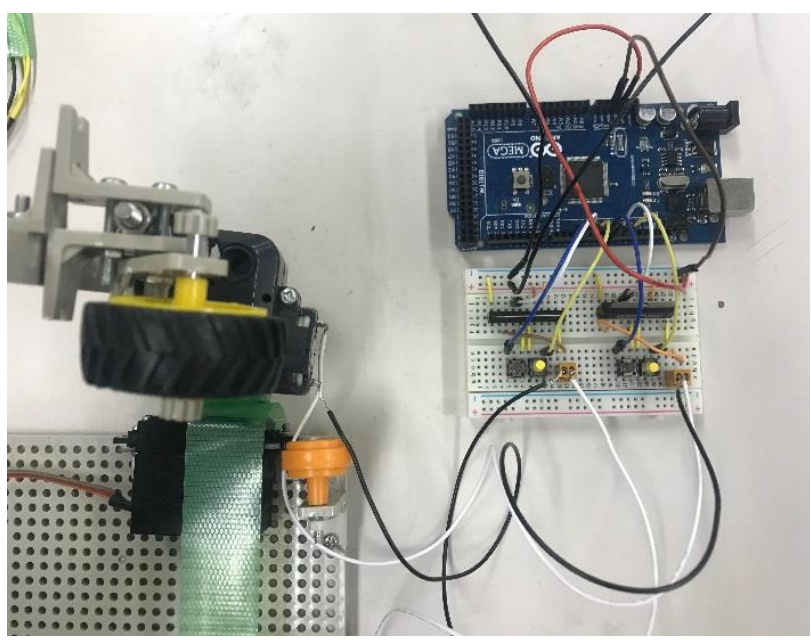

Fig. 2. Servo motor mechanism

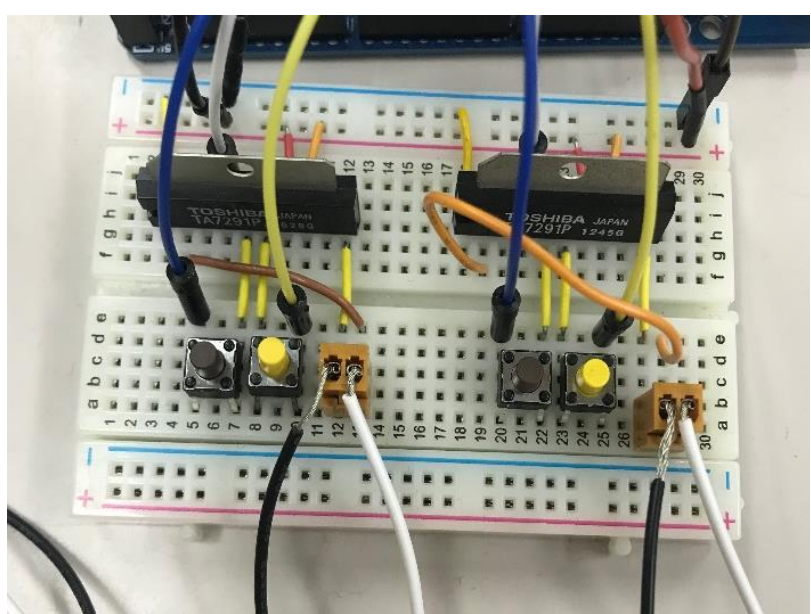

Fig. 3. Switch diagram (for motor)

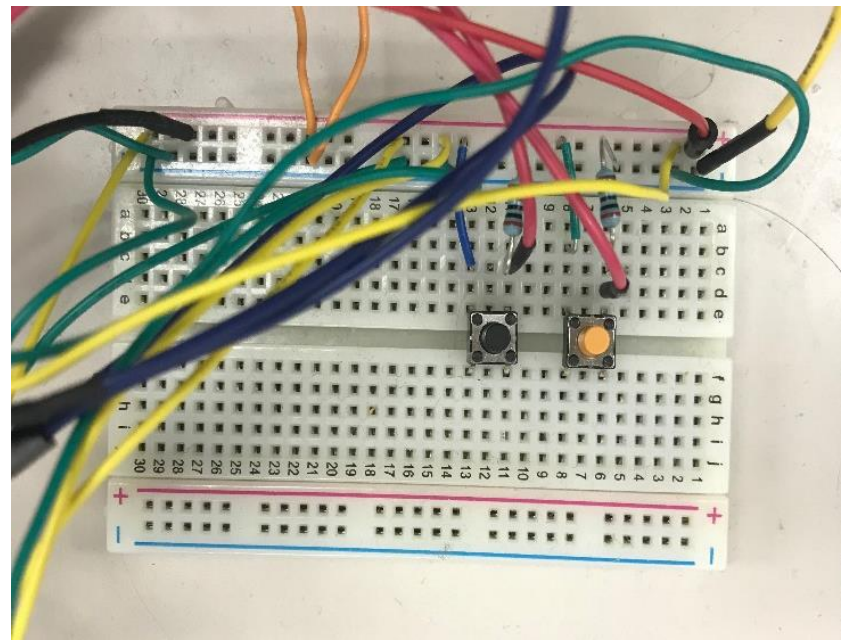

Fig. 4. Switch diagram (for assembly and folding)

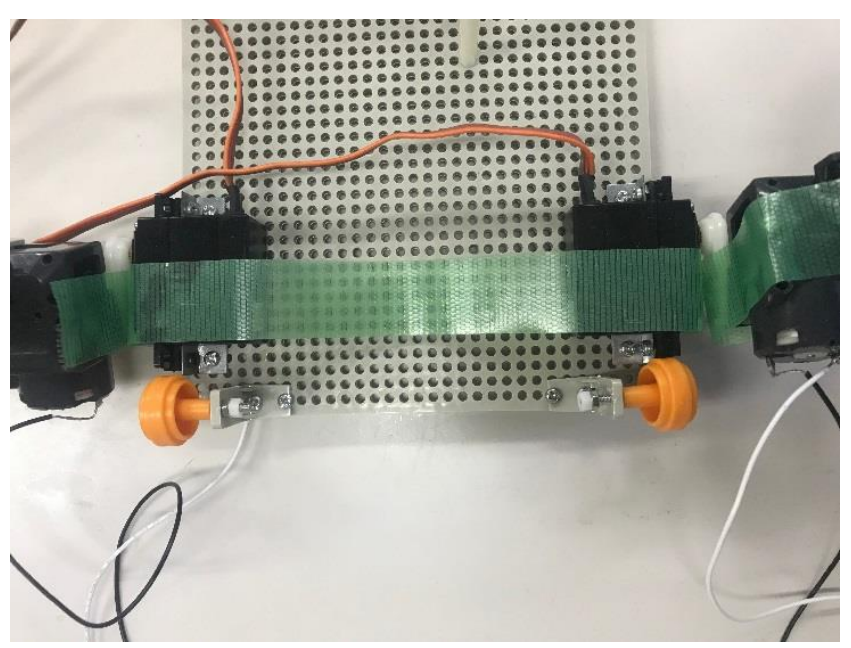

Fig. 5. Table edge tire

Only one Arduino can't controls five servomotor attached to legs and the node of the table. So Arduino and Arduino MEGA were attached respectively.

The table switch is a set of ON button and OFF button. There are two types of switches. One switch starts assembling and folding the table and shown in Fig 3. Another switch starts to move the motor and shown Fig 4. This button is a pair of a button that rotates the motor in the forward direction and a button that rotates the motor in the reverse direction. When the button that rotates the motor in the forward direction is pressed, the legs of the table extend. When you press the button that turns the motor in the reverse direction, the table legs are retracted.

The tires at the end of the table are shown in Fig. 5. The orange attached below the servomotor is the tire. If the tire is not attached, the part may be dragged during the 
assembly and folding operation of the table, but the attachment of the tire enables the operation to be performed without the end being dragged.

\section{Mechanisms}

Next I will explain the mechanism. First I explain the angles of the joints of the legs when the table is assembled and the angles of the joints of the legs when the table is folded. The angle of the joint of the leg when the table is folded is 0 degrees as shown in Fig. 6, and it is folded so that the leg and the plate of the table are parallel. The angle of the leg joints when assembling the table is 90 degrees as shown in Fig. 7 and legs and table board are vertical.

Next, we will explain the mechanism of the joints of the table when assembled and folded. The angle of the joints of the table when the table is folded is as shown in Fig.8. This state is assumed to be at an angle of 0 . When the table is folded, as shown in Fig. 8, it initially stays stands vertically to the ground. The angles of the joints of the table when the table is assembled are as shown in Fig9. From the state where the table is placed vertically, the table plates are opened 90 degrees to the left and right respectively, and the state is opened 180 degrees in total.

Finally I explain the mechanism of the motor attached to the legs of the table.A diagram of the motor is shown in Fig 10. Pressing the button to rotate the motor in the forward direction extends the legs of the table, and pressing the button to rotate the motor in the reverse direction shrinks the legs of the table. The results are shown in Fig 11 and Fig 12.

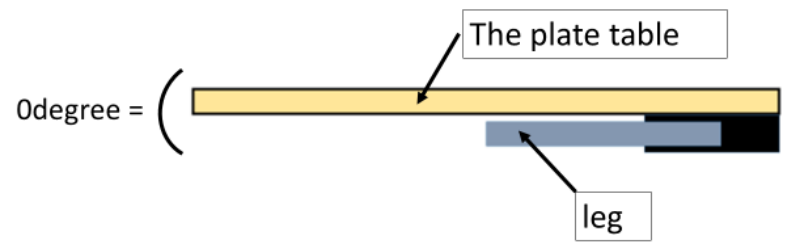

Leg and the plate table are parallel

Fig. 6. Joint angle of the leg when the table is folded

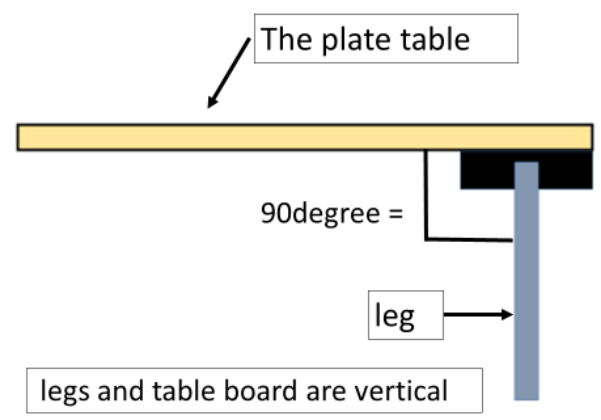

Fig. 7. Joint angle of the leg when assembling the table

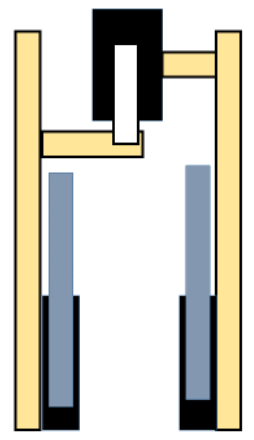

Fig. 8. Joint angle of table when the table is folded

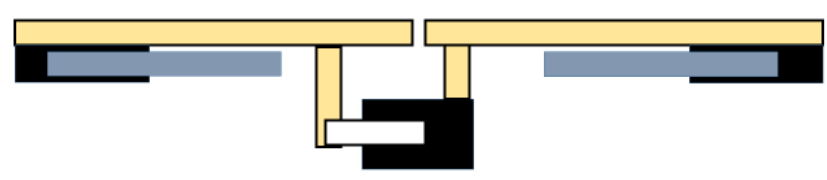

Fig. 9. Joint angle of table when assembling table 


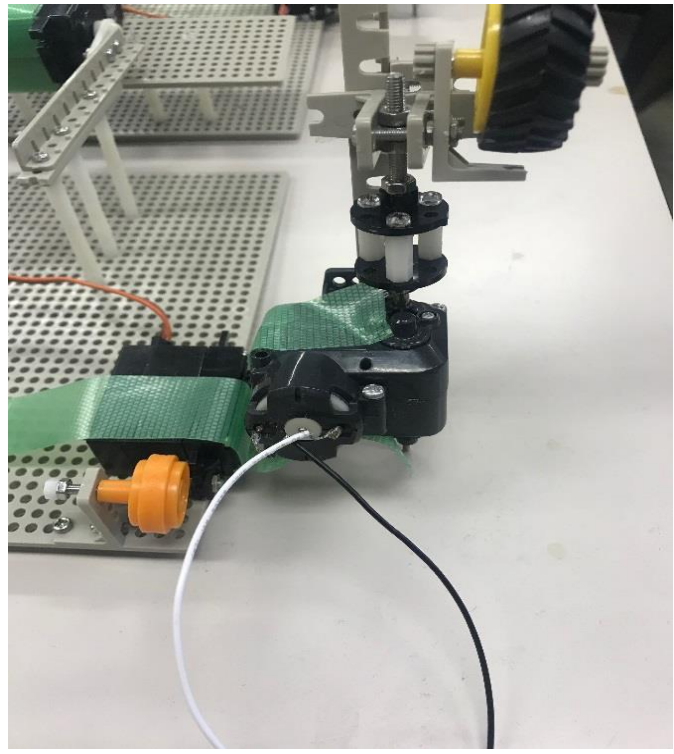

Fig. 10. Table leg motor

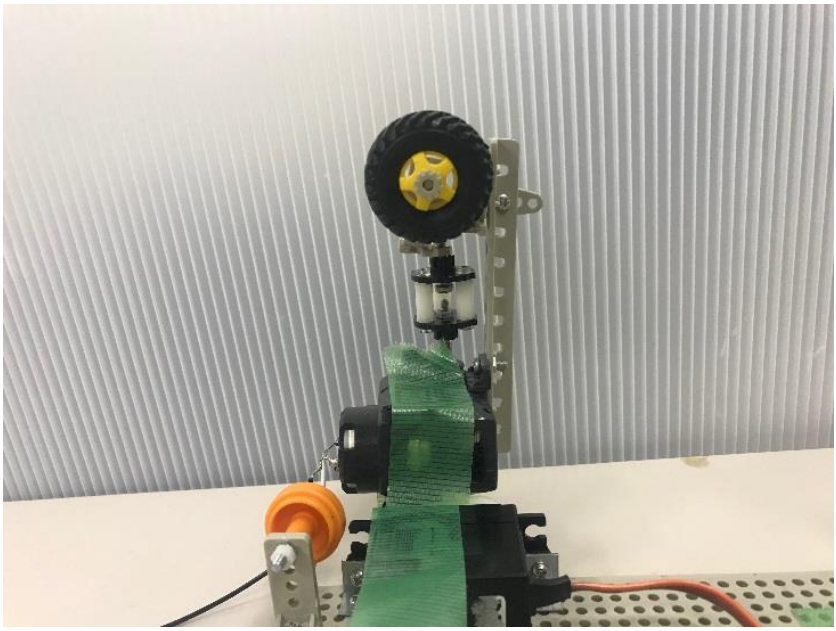

Fig. 11. Motor (when contracted)

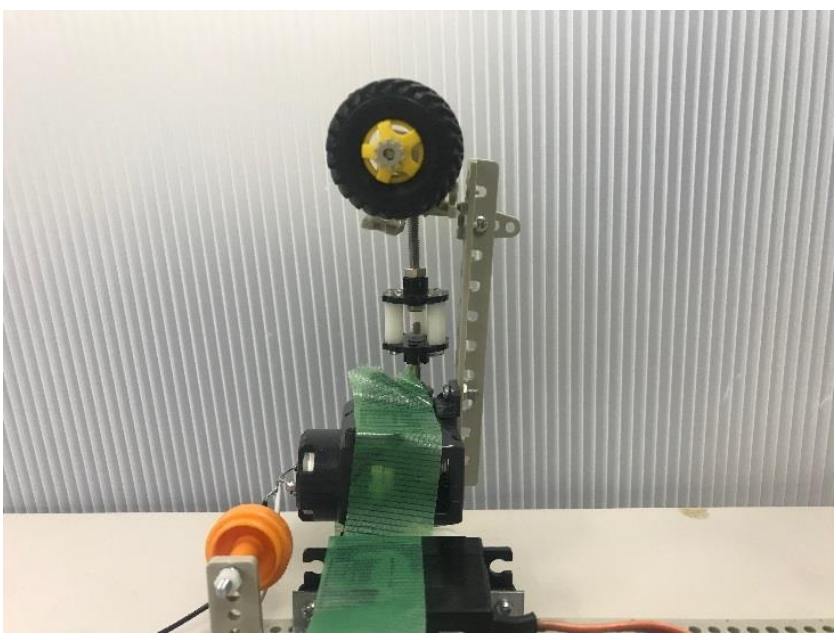

Fig. 12. Motor (when extended)

\section{Explanation of operation}

I will go into the actual operation explanation. The switch of the sensor unit has two switches: one to start the operation to fold the table and the other to open the table. First, let's look at the flow when pressing the switch to open the table.

(1) Press the switch.

(2) The table board opens.

The table opens from the closed position of the plate as shown in Fig. 13 while keeping vertically to the ground. A series of movements are shown in Fig. 14, Fig. 15 and Fig. 16.

(3) The joints of the three legs open.

The joints move as shown in Fig. 17, Fig. 18, Fig. 19 and Fig. 20.The four joints open simultaneously from $0^{\circ}$ to $90^{\circ}$.

Next, let's look at the flow when pressing the folding switch.

(1) Press the switch

(2) Two legs are folded.

(3) Table board closes

The motion is the reverse motion of opening.

To easy understanding shown table movement Figures. Fig 21 shows the movement of the table. And Fig 22 shows the movement of the legs.

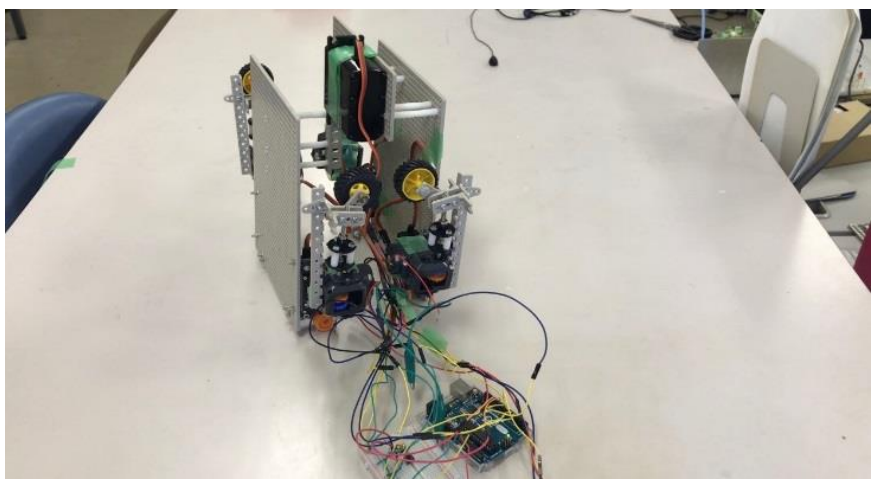

Fig. 13. Table board movement 1 


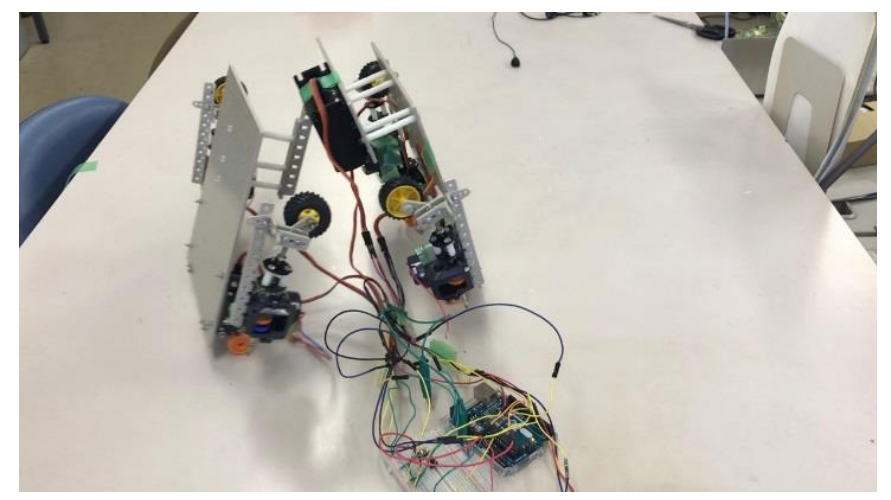

Fig. 14. Table board movement 2

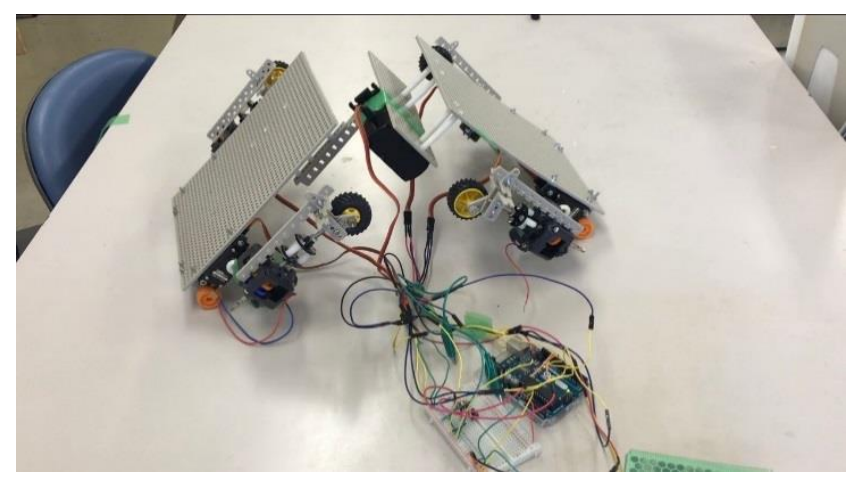

Fig. 15. Table board movement 3

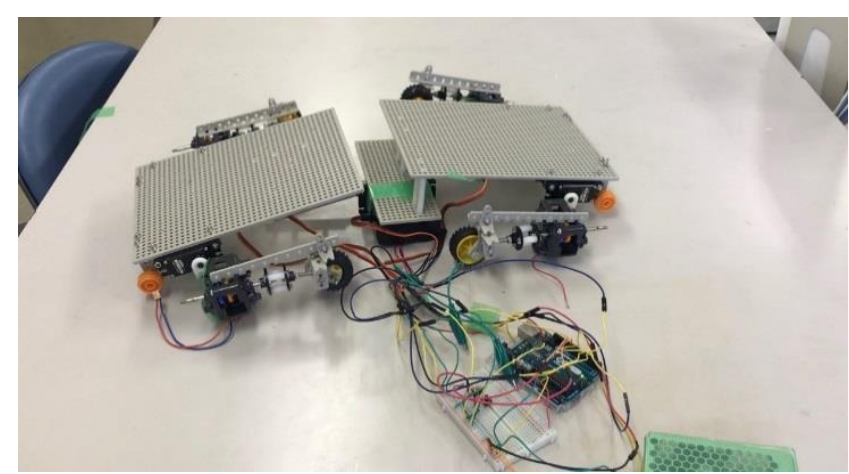

Fig. 16. Table board movement 4

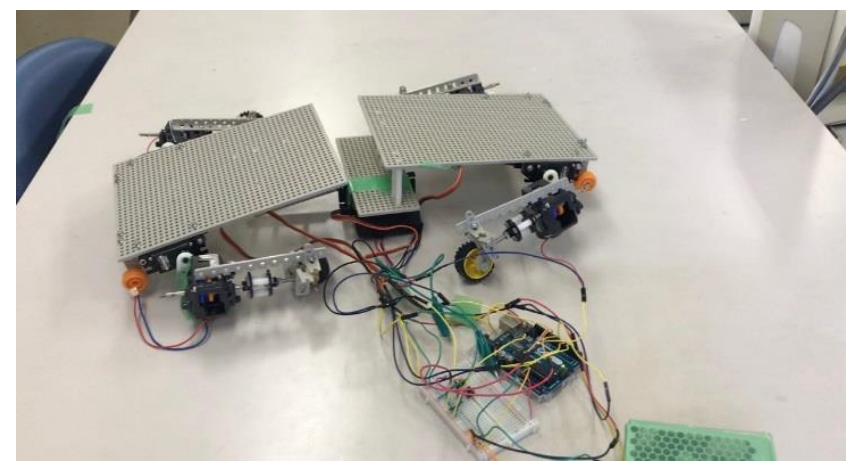

Fig. 17. Leg movement 1

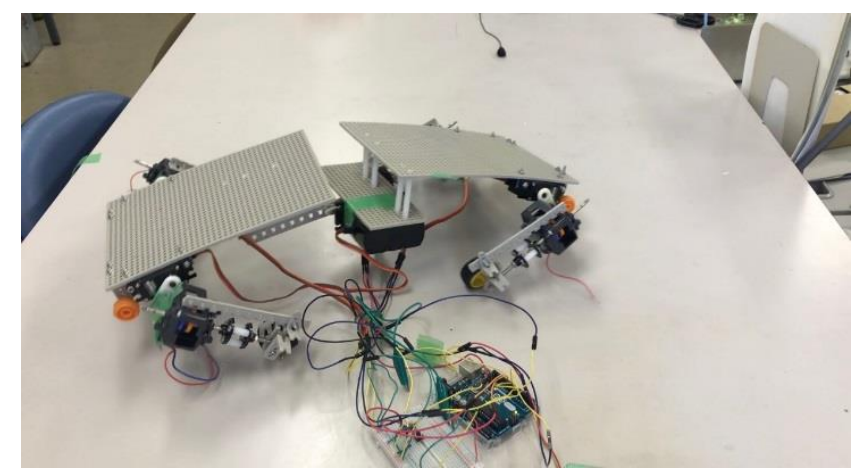

Fig. 18. Leg movement 2

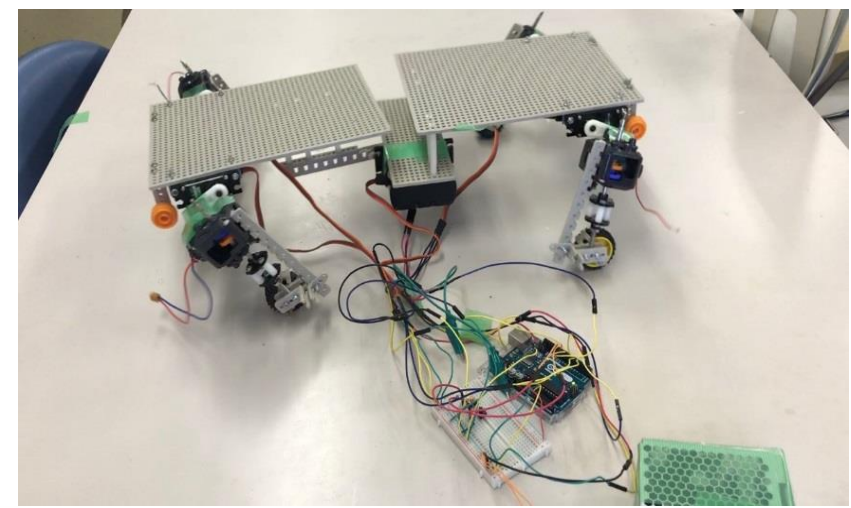

Fig. 19. Leg movement 3

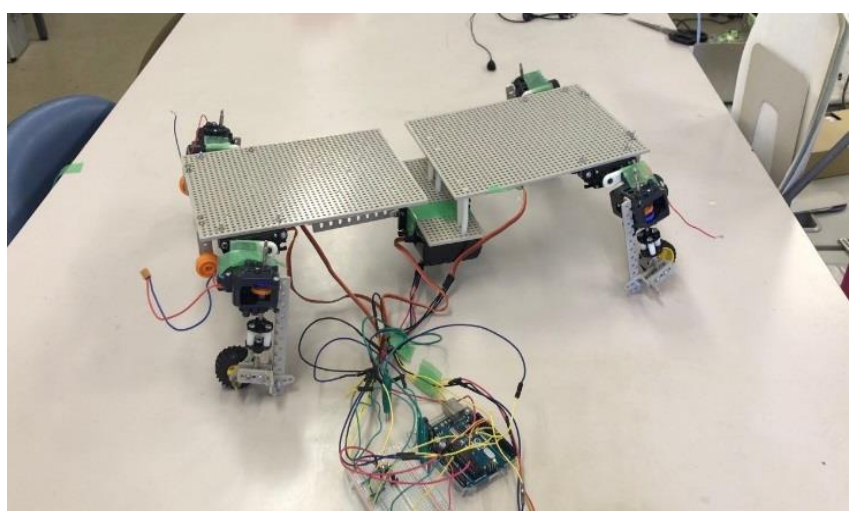

Fig. 20. Leg movement 4

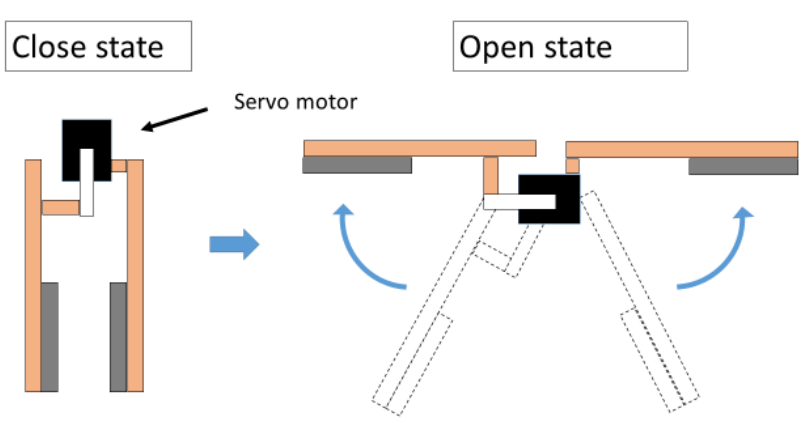

Fig. 21. Table board movement (diagram) 


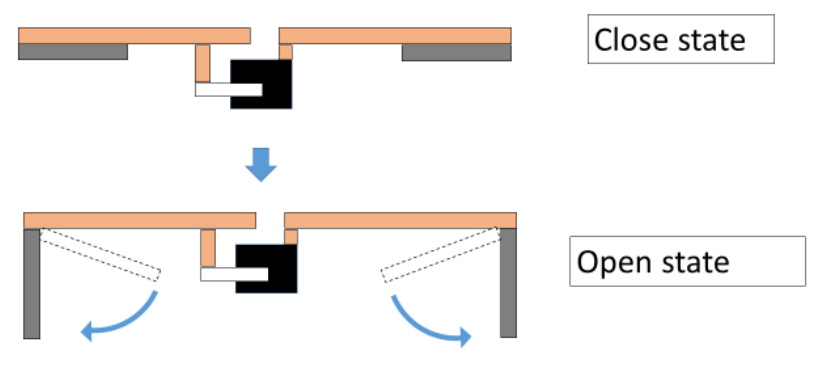

Fig. 22. Leg movement (diagram)

\section{Conclusions}

As a result of the experiment, it was possible to perform the folding and assembling operations. The extension and contraction of the leg was made possible manually by the switch. The table used in this study used a small model. It was not possible to automatically extend and contract the table legs.

It was also found that the performance of the table was impaired because each part of the table was loose. Future issues include automatic leg stretching and strengthen each part of the table.

\section{References}

(1) Takuya Nakanishi, Kanta Aoki, Fumihiro Sakemi, and Mai Fukunaga : "Development of the wooden furniture which assumed office use", Proceeding of the annual conference of JSSD, Vol.62, pp.101, 2015

(2) Cabinet Office

http://www8.cao.go.jp/shougai/whitepaper/h25hakusho /gaiyou/h1_01.html

(3) Masakazu Yamauchi, Hachiro Nishioka and Shiro Koike : "Population Association of Japan", Vol.42, pp.78-, 2008 